\title{
Cold atmospheric single plasma jet for RONS delivery on large biological surfaces
}

\author{
Azadeh Valinataj Omran ${ }^{1}$, Giovanni Busco' ${ }^{2}$, Loïck Ridou ${ }^{2}$, Sébastien Dozias ${ }^{1}$, Catherine Grillon ${ }^{2}$, Jean-Michel \\ Pouvesle $^{1}$ and Eric Robert ${ }^{1}$
}

${ }^{1}$ GREMI, UMR 7344 CNRS/Université d'Orléans, 45067 Orléans, France

${ }^{2}$ Centre de Biophysique Moléculaire, UPR4301CNRS, 45071 Orléans, France

E-mail: azadeh.valinattajomran@univ-orleans.fr and eric.robert@univ-orleans.fr

Received xxxxxx

Accepted for publication $\mathrm{xxxxxx}$

Published xxxxxx

\begin{abstract}
Several attempts have been made to deliver reactive oxygen and nitrogen species (RONS) produced by non-thermal plasma onto large surfaces in controllable way compatible with skin treatments. In this paper, the effect of pulse frequency, in the range 0.5 to $20 \mathrm{kHz}$, on the discharge behavior was studied on skin tissue models to evaluate potential treatment changes. This has been done through electrical characterization, visualization of the helium flow (by Schlieren technique), produced plasma jet modifications (ICCD imaging) and RONS measurements. The results show that, in addition to its well-known important role in the production of the chemical species, the applied discharge frequency plays a very significant role on the size of the treated surface. An enhancement of $\mathrm{NO}^{*}, \mathrm{OH}^{*}$ and $\mathrm{O}^{*}$ production in the gas phase at the higher frequency is reported and assigned to the stronger mixing of the helium flow with ambient air. The efficacy of plasma jet on transporting RONS on/into agarose gel and pig skin has been evaluated. The distribution of the reactive species on the target or passing through, is strongly depending on the discharge frequency and consequently induces $\mathrm{pH}$ variations. The present study supports a new way for enlarging the treated surface by using a simple jet at high frequency in the $20 \mathrm{kHz}$ range leading, with appropriate gas flow and distance to target, to conditions of RONS production that are compatible with potential uses for biomedical or cosmetic applications.
\end{abstract}

Keywords: plasma jet, high repetition rate, RONS delivery, agarose gel, pig skin, biomedical application, cosmetic

\section{Introduction}

Cold atmospheric plasmas represent a great opportunity in plasma research due to their vast potential for biomedical applications and processing of heat-sensitive materials. The potential activities for such plasmas in the biomedical field range from the study of wound healing [1,2], cancer treatment [3-5], dentistry [6-9], sterilization and decontamination [10,
11], dermatology and cosmetics [12-15]. The development of dedicated plasma devices for targeted applications is one of the key aspects that contributes to the rapid advancement of plasma technologies in the biomedical field, besides the studies dedicated to the determination of the mode of action of plasma in interaction with tissues. 
Among others, atmospheric pressure plasma jets are increasingly used in biomedical applications $[16,17]$. These devices enable the generation of a discharge that transports reactive species away from the powered electrode in capillarybased set-up. Helium based plasma jets are very efficient to generate and control non-equilibrium plasmas at atmospheric pressure. Compared to some other plasma reactor types, they allow a quite easy control of stable low temperature plasma plumes as they can operate at much lower voltages than in air, and they are mainly confined within the helium flow outside the capillary due to the higher ionization efficacy than the surrounding air in the used range of electric field. Nevertheless, for reasons that largely have to do with minimizing gas heating and applied voltages, the diameter of the capillary needed to generate an individual plasma jet is typically less than a few mm which then limits the size of the treated area. Development of a large linear or volumetric source of diffuse cold plasma at atmospheric pressure to adapt to the needs of large-scale applications remains an essential challenge both from a scientific and from a technological point of view. Some recent reports have proposed to use arrays of atmospheric pressure plasma jets to increase the area being treated [18-21]. However, since the individual plasma plumes generated by the arrayed plasma jets remain of small size, possibly of high current density, local hot spots still may be delivered by such devices. In this paper, we will present an alternative solution to treat a large area with a single plasma jet in a controllable way. We will show that the pulse repetition frequency can be used as a control parameter for surface RONS delivery to tissue surfaces and underlying layers.

The pulse frequency is also an important controlling factor in the production of the different RONS by atmospheric pressure plasma jets. At low frequency operation, RONS are largely flowing out of the plasma production zone prior to the next discharge pulse favoring production of primary RONS. Conversely, at high frequency, pulse accumulation may result in a difference RONS balance. This may favor the generation of secondary RONS that require several collisions with other RONS to be generated [22]. Once the RONS are delivered to the surface, their availability deeper in the tissue remains one of the key points to study. Over the last few years, there has been a considerable amount of experimental work aimed at understanding the RONS production and their penetration and distribution on/into the tissues. To better understand plasmatissue interactions, some simple models have been developed. Among these models, agarose gel is commonly used to mimic different tissues mainly for its physical properties [23]. This gel was used by Szili et al. to study the penetration of RONS generated by plasma jets. Their results show that the plasma can induce the transport of RONS into the agarose gel and that the plasma exposure time, agarose film thickness and the distance between the plasma source to the target influence the amount and rate of RONS delivery [24]. Kawasaki et al. used a KI-starch reagent (iodine test) to visualize the twodimensional distribution of ROS over a tissue model also made of agarose gel [25]. Recently, it has been shown that plasma jets deliver RONS through millimeter-thick real biological tissue both in vitro (pig muscle) [26] and in vivo (cancer tumor embedded underneath mouse skin) [27]. In addition, it must be mentioned that the tissue physical and chemical properties such as roughness, humidity, wettability, and conductivity together with the gap distance (i.e., distance between the tip of the capillary and the target) and the pulse repetition frequency can themselves influence the plasma treatment [28]. The operating conditions and the target features induce feedback on the plasma characteristics such as concentration of reactive species and gas temperature, which can in turn change the effectiveness, distribution and penetration of the plasma on/in the tissue.

As the present work is in perspective of the development of plasma for skin treatments in applications ranging from medicine to cosmetics [29], the two relevant topics investigated here are the large surface plasma delivery mechanisms and the interactions with tissue models mimicking human skin. The materials and methods are presented in section 2 . The results and discussion are detailed in section 3. Concluding remarks and perspective will be given in section 4.

\section{Materials and Methods}

In this section, we will present the plasma reactor and experimental setup, plasma diagnostic methods, the tissue model and chemical analysis for RONS measurement. Different plasma jet operational conditions such as discharge frequencies and gap (distance from the capillary outlet to the target) were used to analyze the effect of plasma jet on RONS production and treated area size.

\subsection{Experimental setup}

The plasma jet used in this work is a plasma gun (PG) device [30]. Figure 1(a) shows a schematic view of the PG. The PG is a coaxial dielectric barrier discharge reactor with a glass capillary flushed with helium. The $12 \mathrm{~cm}$ long capillary was tapered at the outlet $\left(\varnothing_{\text {in }}=1.5 \mathrm{~mm}, \varnothing_{\text {ext }}=3 \mathrm{~mm}\right)$. The helium flow rate through the reactor capillary was $0.5 \mathrm{slm}$. In this work, the PG device was driven by $\mu$ s duration voltage pulses (positive polarity) applied to a hollow inner electrode. Grounded ring electrode was set outside the capillary at the level of the inner electrode tip. The high voltage was set at 9 $\mathrm{kV}$. The applied voltage was measured using a high-voltage probe (Tektronix P6015A). The discharge current through the agarose gel target has been measured using a $100 \Omega$ shunt resistor connected in series with the target. Experiments were performed at different frequencies $(0.5,2,20 \mathrm{kHz})$ and gap distances $(10,15,20 \mathrm{~mm})$. The discharge power has been 
measured from V-Q Lissajous figures obtained using a $1.5 \mathrm{nF}$ capacitor connected in series to the grounded electrode or grounded target. Target temperature during the treatment was measured using an infrared camera (FLIR E5). The temperatures were measured after two minutes treatment. The plasma emission was measured using a $1 \mathrm{~mm}$ diameter optical fiber located downstream at $5 \mathrm{~mm}$ from the capillary exit and connected to the Ocean Optics spectrometer (Maya 2000 Pro), sensitive from 200 to $1100 \mathrm{~nm}$. The optical fiber was positioned horizontally $7 \mathrm{~mm}$ away from the plasma jet axis.

Plasma spectra were collected by averaging 100 exposure gates, each being of $800 \mathrm{~ms}, 200 \mathrm{~ms}$ and $20 \mathrm{~ms}$ at $0.5 \mathrm{kHz}, 2$ $\mathrm{kHz}$ and $20 \mathrm{kHz}$, respectively. The helium flow at the capillary exit was visualized using a Schlieren optical bench in $\mathrm{Z}$ type configuration as sketched in figure 1(b). The parabolic mirrors were $20 \mathrm{~cm}$ in diameter and have a focal distance of $1 \mathrm{~m}$. The light source was a green light diode. A fast CCD camera (IDTStreal XS-3) was used to visualize the helium flow expansion in the ambient air as in previous work [31]. Time resolved plasma plume propagation downstream of the capillary exit was visualized through ICCD imaging (PIMAX3 ICCD camera), synchronized on the voltage pulse onset.

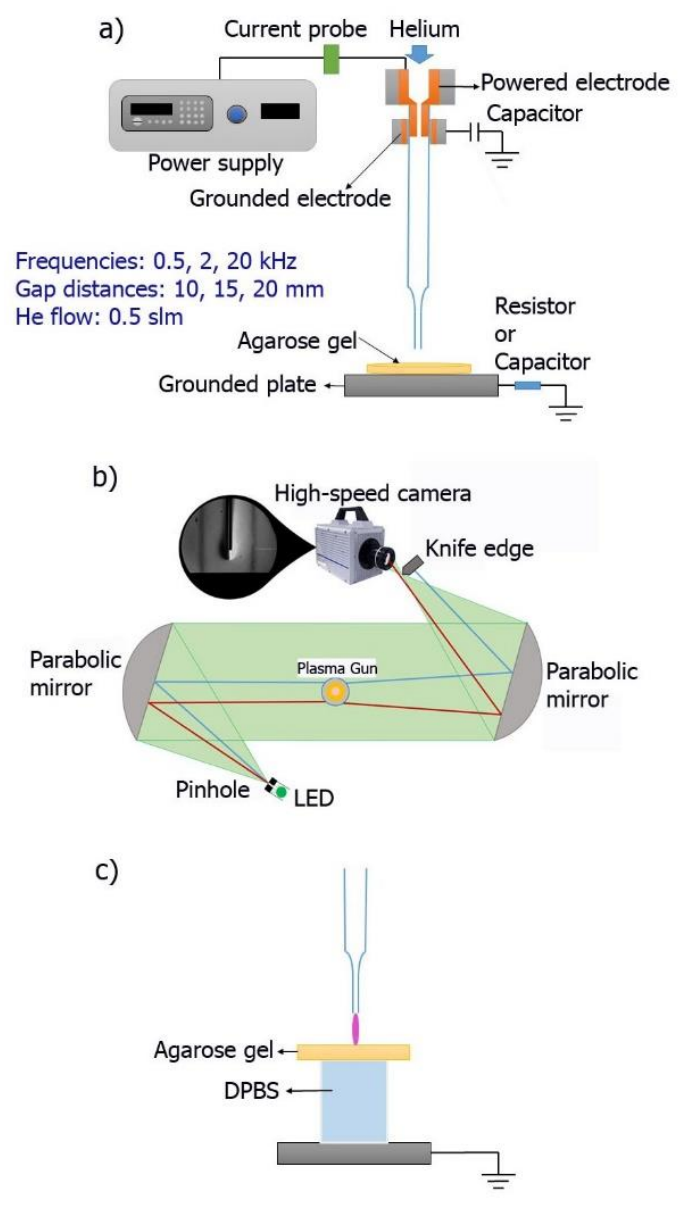

Figure 1. a) Experimental setup, b) Optical Schlieren bench in Z type configuration and c) RONS delivery setup.

\subsection{Reagents}

Dulbecco's modified Phosphate Buffered Saline (DPBS) was from GIBCO. Soluble starch was from Prolabo. TopVision agarose and Amplex ${ }^{\mathrm{TM}}$ Red Hydrogen Peroxide/Peroxidase Assay Kit were from Thermo Fisher Scientific. Potassium iodide (KI), sodium chloride $(\mathrm{NaCl})$, sodium nitrite $\left(\mathrm{NaNO}_{2}\right)$, agarose for microbiology, $\mathrm{N}-(1$ naphthyl) ethylene-diamine dihydrochloride (NEDD) and sulfanilamide were from Sigma Aldrich.

\subsection{Preparation of agarose gel targets}

The $1.5 \%(\mathrm{w} / \mathrm{v})$ agarose gel target was prepared by dissolving $1.5 \mathrm{mg} / \mathrm{mL}$ agarose for microbiology powder in a physiological saline solution $(\mathrm{NaCl} 150 \mathrm{mM})$. The solution was heated on a conventional heating magnetic stirrer until all the powder was dissolved. Agarose mix was poured to obtain gels of $2 \mathrm{~mm}$ thickness. The gels were stored at $4^{\circ} \mathrm{C}$ for 12 hours before use.

KI-starch-loaded agarose gel was used to evaluate the spatial distribution of PG generated ROS. The samples were prepared by dissolving $1.5 \%$ agarose for microbiology powder, $0.3 \%$ potassium iodide and $0.5 \%$ starch $\left(\left(\mathrm{C}_{6} \mathrm{H}_{10} \mathrm{O}_{5}\right)_{\mathrm{n}}\right)$ in a saline physiological solution $(\mathrm{NaCl} 150 \mathrm{mM})$. The KIstarch reagent can react with hydroxyl radicals $\left({ }^{\circ} \mathrm{OH}\right)$, atomic oxygen $\left(\mathrm{O}^{\bullet}\right), \mathrm{H}_{2} \mathrm{O}_{2}$, and $\mathrm{HO}_{2}$ radicals [32].

\subsection{RONS diffusion through agarose gel}

The $2 \mathrm{~mm}$ thick agarose gels were cut in squares of $15 \times 15$ $\mathrm{mm}^{2}$ placed on the top of wells of 96-well plates completely filled with DPBS. Agarose gels laid on the 96-well plates were then plasma treated. The schematic setup is shown in figure 1 (c). Fifteen minutes after treatment, DPBS samples were collected from the well and transferred in new 96-well plates to measure the amount of hydrogen peroxide, nitrites or RONS passed through the agarose gels.

To study the potential influence of the plasma on the RONS penetration during the plasma application, a second set of experiments has been performed. After $120 \mathrm{~s}$ treatment above DPBS filled wells, the agarose gels were immediately transferred to another well plate with fresh DPBS filled wells. Then RONS were measured in both DPBS filled wells, the first one immediately after the treatment and the second 15 min after the gel transfer. RONS measurement were performed in triplicate for each treatment condition. These data are shown as the mean and deviations for the three individual measurements.

\subsection{Hydrogen Peroxide measurement}


The concentration of hydrogen peroxide $\left(\mathrm{H}_{2} \mathrm{O}_{2}\right)$ was measured with the Amplex ${ }^{\mathrm{TM}}$ Red Hydrogen Peroxide/Peroxidase assay kit (Thermofisher Scientific). The Amplex ${ }^{\mathrm{TM}}$ Red reagent is converted in the red-fluorescent resorufin in presence of $\mathrm{H}_{2} \mathrm{O}_{2}$ and peroxidase (HRP). After each treatment, a volume of $50 \mu \mathrm{L}$ of DPBS was collected and transferred into individual wells of a 96-well plate. Standard $\mathrm{H}_{2} \mathrm{O}_{2}$ solutions $(0-70 \mu \mathrm{M})$ were prepared in DPBS to make a calibration curve and $50 \mu 1$ of each standard were transferred in the 96 well plate. A working solution of $100 \mu \mathrm{M}$ Amplex ${ }^{\mathrm{TM}}$ Red reagent and $0.2 \mathrm{U} / \mathrm{mL}$ HRP were prepared following the manufacturer's protocol. Then, $50 \mu \mathrm{L}$ of the working solution were added to each well containing the standards, the controls and the samples. The 96-well plate was incubated in the dark at room temperature for $30 \mathrm{~min}$. Then the emitted fluorescence intensity was measured by a microplate reader spectrofluorometer (Victor 3V, PerkinElmer), excitation wavelength at $531 \mathrm{~nm}$ and emission wavelength of $605 \mathrm{~nm}$.

\subsection{Nitrite measurement}

A Griess test was used to measure the concentration of nitrites passed through the agarose gel after treatment. $\mathrm{NaNO}_{2}$ standard solutions $(0-100 \mu \mathrm{M})$ were prepared in DPBS to make a calibration curve. $40 \mu \mathrm{l}$ of controls, standards and samples were transferred in a 96-well plate containing $80 \mu 1$ of sulfanilamide ( $3 \mathrm{mM}$ in $30 \%$ acetic acid) and $80 \mu \mathrm{l}$ of NEDD $(0.2 \mathrm{mM}$ in $60 \%$ acetic acid). After $10 \mathrm{~min}$ of incubation, absorbance was measured at $560 \mathrm{~nm}$ with the microplate reader spectrofluorometer (Victor3 V, PerkinElmer).

\subsection{Agarose gel surface pH measurement}

Spatial distribution of the acidic species and $\mathrm{pH}$ measurement were assessed using the protocol derived of a previously published method [33]. In this work, acetic acid/acetate buffer was used instead of HEPES buffer. Briefly, five acetic acid/acetate buffers $(50 \mathrm{mM})$ containing $100 \mathrm{mM}$ $\mathrm{NaCl}$ were prepared with $\mathrm{pH}$ ranging from 5.5 to 3.6, following the Henderson-Hasselbalch approximation. Precise $\mathrm{pH}$ of each buffered solution was measured with a $\mathrm{pH}$ meter. $50 \mu \mathrm{M}$ of fluorescein sodium salt and $2 \%$ of TopVision Agarose (Thermofisher) were added to each buffered solution. The resulting mixtures were gently heated in a microwave and poured in 6-well plates. The volume was calculated in order to get a $2 \mathrm{~mm}$ thick gels in each well. The gel at pH 5.5 was used as a skin phantom model. Plasma treatment was performed using PG at different frequencies and fluorescence of each agarose-containing well was measured using the Typhoon ${ }^{\mathrm{TM}}$ FLA 9500 biomolecular imager (GE Healthcare Life Science) equipped with a $437 \mathrm{~nm}$ (blue LD laser) and a Y520 emission filter (wavelength range $\geq 520 \mathrm{~nm}$ ). Fluorescein fluorescence analysis was performed using the Image-J software.

\subsection{KI-starch test on pig ear skin discs}

To visualize the ROS distribution on the skin surface, we used discs of $35 \mathrm{~mm}$ in diameter cut in fresh pig ears. They were soaked in a solution of $150 \mathrm{mM} \mathrm{NaCl}, 0.3 \% \mathrm{KI}$ and $0.5 \%$ starch for two hours and then treated with PG at various frequencies. ROS distribution on skin surface was visualized by the dark purple color generated by the KI-starch/ROS reaction. Pictures of the samples were taken before and after each treatment. It must be pointed out that a homogenous penetration and distribution of the above mentioned solution is not fully guaranteed in the pig ear skin after soaking. The coloration of the pig ear must then be considered more as an indicator of the RONS delivery than a real measurement.

\section{Results and discussion}

\subsection{Plasma characteristics}

The waveforms of applied voltage and total current of the plasma jet at $2 \mathrm{kHz}$ are presented in figure $2 \mathrm{a}$. The pulse is independent of the pulse frequency for the PG operated with our power supply. The calculated applied power on the high voltage electrode from figure $2 \mathrm{a}$ is $0.25 \mathrm{~W}$. As can be seen, the gap distance between nozzle and grounded electrode has little influence on the total current but this was not anymore true for the current though the agarose gel target. For example, increasing the gap distance from 10 to $20 \mathrm{~mm}$ results in a decrease of more than $20 \mathrm{~mA}$ of the peak current through the target (figure 2b). At the frequencies of $0.5,2,20 \mathrm{kHz}$, the average current through the target is $0.012,0.05$ and $0.5 \mathrm{~mA}$, respectively. These values are well below the recommended requirements to avoid the electric shock and below the threshold of current perception around $1 \mathrm{~mA}$ [34].

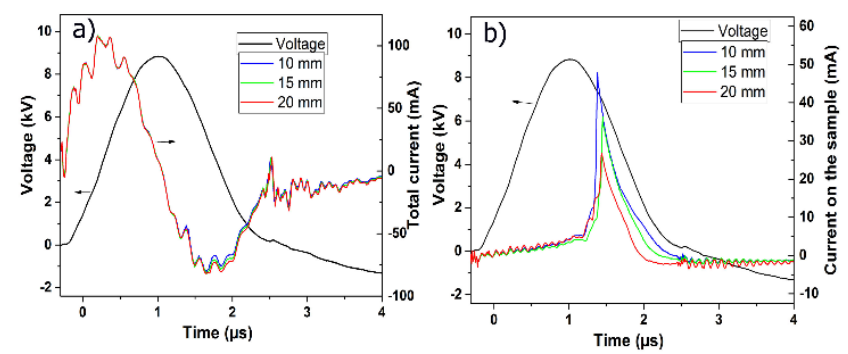

Figure 2. Voltage pulse applied on the electrode a) total current, b) current time evolution measured across the target with a $100 \Omega$ resistor. Applied frequency: $2 \mathrm{kHz}$ and He flow: $0.5 \mathrm{slm}$.

The method commonly employed for obtaining the discharge power is based on the Q/V Lissajous figure. The area of the closed V-Q loop for one period of the applied voltage is equal to the energy dissipated per one cycle. The mean discharge power is then simply the energy per cycle multiplied by the working frequency. The Q/V Lissajous curves of the plasma jet are shown in figure 3. for an applied 
voltage amplitude of $9 \mathrm{kV}$ at $2 \mathrm{kHz}$ and a gas flow of $0.5 \mathrm{slm}$ for three gap distances of $10 \mathrm{~mm}, 15 \mathrm{~mm}$ and $20 \mathrm{~mm}$ between capillary exit and the target. On the one hand, for these operating conditions, the obtained Lissajous curves on the grounded electrode were almost similar for all the gap distances (figure 3a). The area and power calculated from the Lissajous curves on the grounded electrode were about $13 \mu \mathrm{J}$ and $26 \mathrm{~mW}$, respectively. On the other hand, as it can be seen in figure $3 b$, there are quite big variations of these $v$

.alues with the gap distance at the target level. Most of the charges passed through the target and the calculated area of the Lissajous curves related to the agarose gel target for 10 $\mathrm{mm}, 15 \mathrm{~mm}$ and $20 \mathrm{~mm}$ gap distances corresponds to $45 \mu \mathrm{J}$, $32 \mu \mathrm{J}$ and $25 \mu \mathrm{J}$ resulting in a power of $90 \mathrm{~mW}, 64 \mathrm{~mW}$ and $50 \mathrm{~mW}$, respectively. As expected, the amount of charge transferred to the agarose gel target during a discharge cycle markedly decreased with increasing gap distance while, as mentioned above, the charge deposition on the grounded electrode was constant. The same values have been measured for the frequencies of $0.5 \mathrm{kHz}$ and $20 \mathrm{kHz}$.
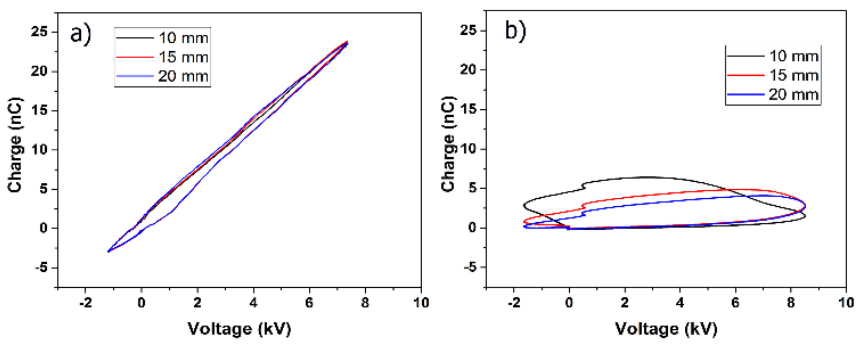

Figure 3. Q/V plots measured a) on the grounded electrode and b) on the agarose gel target for different gap distances.

As the frequency is a crucial parameter in plasma generation, its effect on the target temperature during plasma treatment for the various gap distances has been investigated. As shown in figure 4, as expected, the agarose gel temperature increases with the frequency and decreases with the gap distance. At the low helium flow $(0.5 \mathrm{slm})$ used in this experiment and with a frequency of $20 \mathrm{kHz}$, the temperature at the shortest distance is too high for skin treatments. But it must be mentioned that the reached temperature reduces to acceptable values, below $30^{\circ} \mathrm{C}$, as soon as the helium flow gets to $1 \mathrm{~s} / \mathrm{m}$. In the following, taking into account the measured plasma jet electrical characteristics and the target temperature, a fixed gap of $15 \mathrm{~mm}$ was chosen for the study of RONS production and their delivery. With these parameters the PG did not physically damage the synthetic and biological tissue model targets (at the macroscopic level).

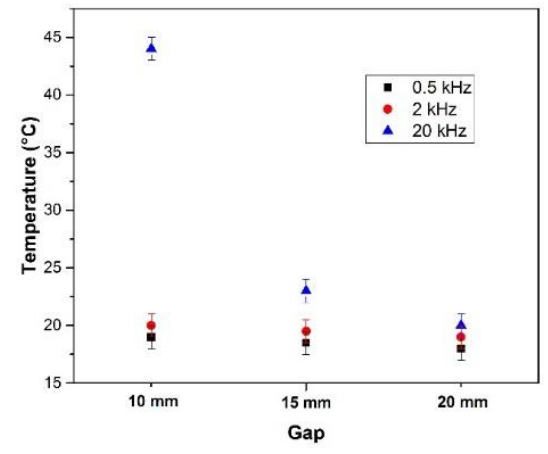

Figure 4. Temperature measurement on the agarose gel target according to the frequency and to the gap distance. He flow: $0.5 \mathrm{slm}$.

To investigate the possible effect of pulse repetition rate on reactive species production in the gas phase, the optical emission from the plasma plume has been analyzed onto an agarose gel target, at the $15 \mathrm{~mm}$ gap distance, using different frequencies (figure 5a). A way to directly observe any changes, without any problem of species quantification, is to use the line ratio intensities of different excited species state to the helium one. Thus we consider that the energy is mainly deposited through helium excitation and ionization while the production of the excited states of the reactive species result from the helium induced kinetics. Figure $5 \mathrm{~b}$ shows the ratios of $\mathrm{I}_{\mathrm{OH}(308)} / \mathrm{I}_{\mathrm{He}: 706 \mathrm{~nm}}, \mathrm{I}_{\mathrm{N} 2+: 391 \mathrm{~nm}} / \mathrm{I}_{\mathrm{He}: 706 \mathrm{~nm}}$ and $\mathrm{I}_{\mathrm{O}(777)} / \mathrm{I}_{\mathrm{He}: 706 \mathrm{~nm}}$ as a function of discharge pulse frequency. As expected, it can be seen the emission ratio $\left(\mathrm{I}_{\mathrm{N} 2+: 391 \mathrm{~nm}} / \mathrm{I}_{\mathrm{He}: 706 \mathrm{~nm}}\right)$ is almost constant as a function of frequency, since the production of the $\mathrm{N}_{2}{ }^{+} \mathrm{B}$ sate at the origin of the transition at $391 \mathrm{~nm}$ results from the direct charge transfer to Penning ionization of $\mathrm{N}_{2}$ from helium ions and excited states respectively. However, the other emission ratios are frequency dependent, especially for $\mathrm{OH}^{*}$ and even more for $\mathrm{NO}^{*}$ excited states for which there is a very strong increase at the highest frequency of $20 \mathrm{kHz}$ indicating probable changes in the mixing of helium flow with ambient air and water vapor arising from the target. That promotion of $\mathrm{NO}^{*}$ production at $20 \mathrm{kHz}$ is of particular interest as $\mathrm{NO}^{*}$ plays an important role in biology and has been shown to regulate many processes in human skin physiology and particularly in blood flow $[35,36]$. These results indicate that the plasma repetition rate influences the variety of reactive species and their relative abundance. 

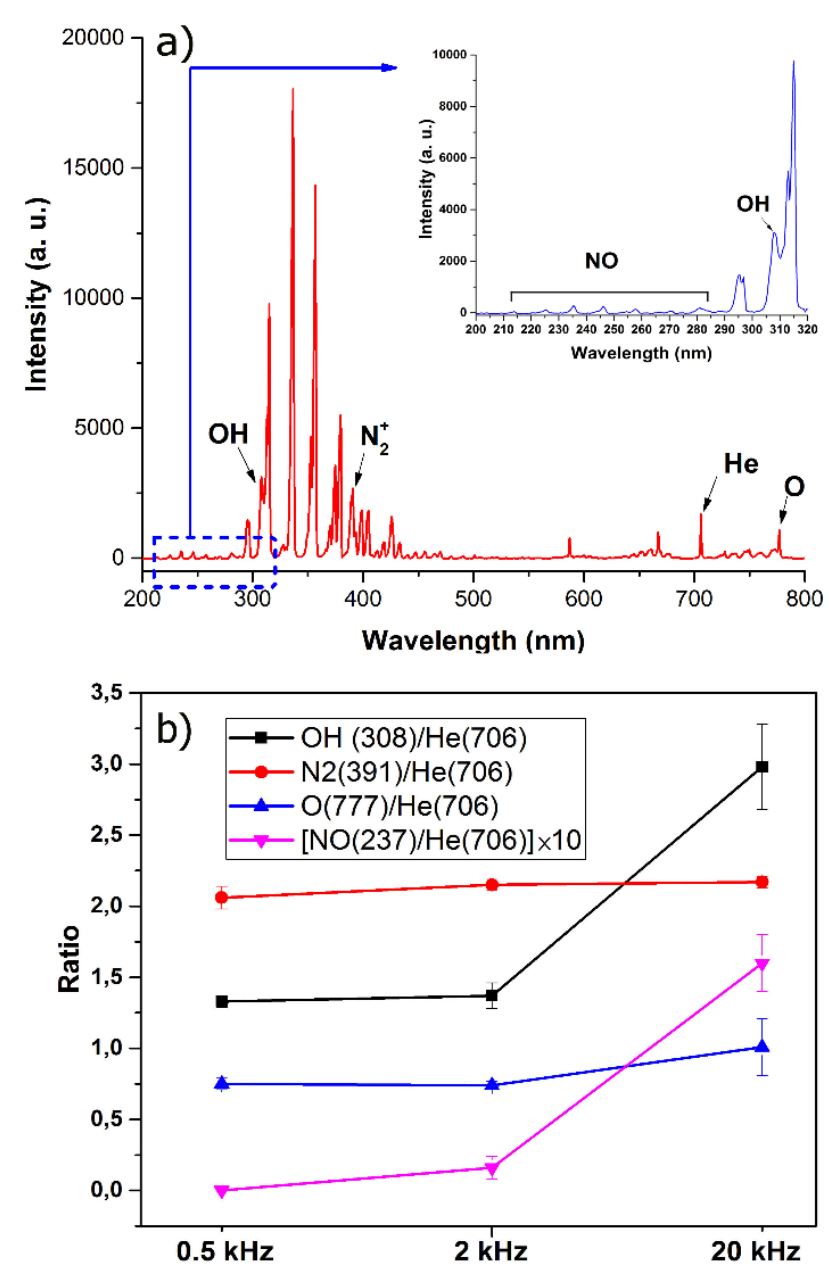

Figure 5. Optical emission of the plasma plume: a) Typical emission spectra of PG in UV region at $20 \mathrm{kHz}$ frequency and b) Ratio of $\mathrm{N}_{2}{ }^{+}$: $391 \mathrm{~nm}, \mathrm{O}^{*}: 777 \mathrm{~nm}, \mathrm{OH}^{*}: 308 \mathrm{~nm}$ and NO*: $237 \mathrm{~nm}$ versus He*: $706 \mathrm{~nm}$ as a function of the frequency. Gap distance: $15 \mathrm{~mm}$; He flow: $0.5 \mathrm{slm}$.

For a better understanding of the role of pulse repetition rate and to complete the OES results, Schlieren technique and ICCD imaging have been used to visualize the behavior of the helium flow and the plasma development, respectively. The results of Schlieren experiments are presented in Figure 6. As can be seen in the series of pictures, the variation of frequency has a strong impact on the way that the helium mixes with air. By increasing the frequency, the mixing zone is largely increased favoring helium plasma transfers to nitrogen, oxygen and water molecules, indeed resulting in significant effects on the density and distribution of the RONS in the effluent of the plasma jet as shown in some previous works $[37,38]$.

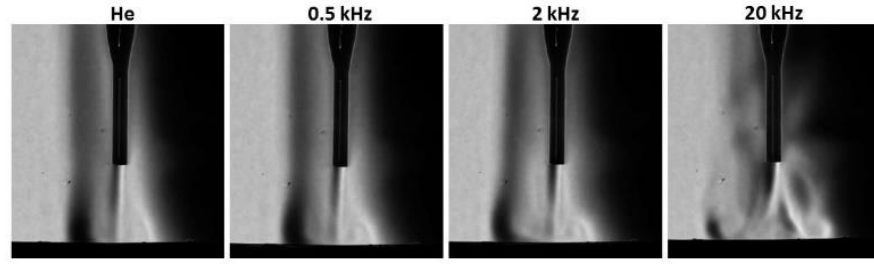

Figure 6. Schlieren images of the plasma jet on the agarose gel target for different frequencies. Gap distance: $15 \mathrm{~mm}$, He flow: $0.5 \mathrm{slm}$.

Figure 7a shows ICCD images of the plasma jet at different frequencies taken by the high-speed camera with an average on 1500 pulses. The discharge conditions were the same as those for the results reported in figure 6 . To better visualize the treated zone, a reduced false color scale has been used (figure 7b). The ICCD imaging observations are in agreement with the previous Schlieren results. Drastic modifications have been observed for the plasma plume behavior and shape as the frequency is increased. At the target surface, instead of a localized plasma spot on the capillary axis obtained at 0.5 $\mathrm{kHz}$ and $2 \mathrm{kHz}$, as already pointed out [31], the generated plasma at $20 \mathrm{kHz}$ ends in a more diffuse and scattered mode and covers a much larger surface. Therefore, the Schlieren and ICCD imaging confirm what was suggested by OES results shown in figure 5 . Changing the frequency from $0.5 \mathrm{kHz}$ to 20 $\mathrm{kHz}$ increases the production of reactive species due to the favorable diffusion of air in the helium flow.

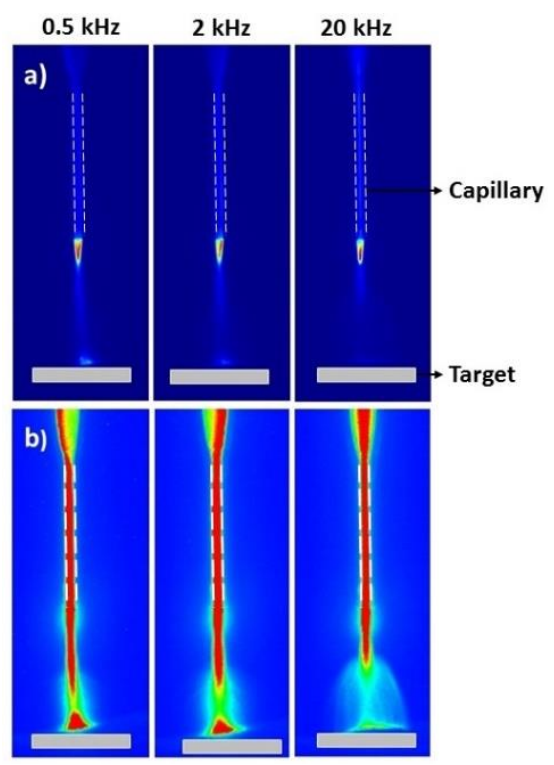

Figure 7. ICCD images of PG for different frequencies with the average on 1500 measurements a) images with full color scale and b) images with reduced false color scale. Gap distance: $15 \mathrm{~mm}$. 


\subsection{RONS delivery on tissue mimicking targets}

Plasma biomedical effects are currently thought to arise mainly from the amount of RONS delivered to cells and tissue [39]. For in vitro treatment on cells covered by a physiological medium, plasma-generated species are delivered in the liquid. This leads to an enriched longer lived reactive species medium and, thus, to a more uniform treatment of the cell monolayer. In the case of skin, the epidermis layer is slightly hydrated and directly exposed to the air. For this reason, in in vivo plasma treatments, the induced biological effects depend not only on the total generated reactive species in the gas phase but also on their distribution and on their density on the surface. To evaluate the ROS spatial distribution and their density on the target surface, we used KI-starch-loaded agarose gels. When exposed to plasma-generated reactive species, the KI-starchloaded agarose gel changed from colorless to blue-purple [40], the color change remaining stable after the treatment. The blue-purple color corresponded to the spatial distribution of ROS on the gel surface, visually indicating the occurrence of oxidation reactions between ROS and KI-starch. In the run experiments, the KI-starch gels have been treated with PG for 120 s. To better visualize ROS distribution and density, the samples were scanned first in black and white and then the images were converted in 8-bit spectrum-gray color. At 20 $\mathrm{kHz}$, as expected from the results presented in figure 7, ROS were spread over a large surface covering in that case the whole surface of $35 \mathrm{~mm}$ diameter petri dish after the $120 \mathrm{~s}$ treatment (figure 8) whereas for 0.5 and $2 \mathrm{kHz}$ the ROS distributions were more focalized in the center.
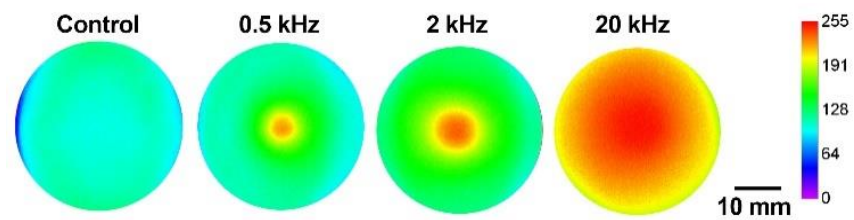

Figure 8. Distribution patterns of ROS on the surface of agarose gels after treatment by the PG for different frequencies using the KI-starch technique. Treatment time: $120 \mathrm{~s}$ and Gap distance: $15 \mathrm{~mm}$.

From an application perspective, it is important to confirm these results by assessing the surface distribution of ROS on real tissues. That has been tested on fresh skin discs from pig ear and results are shown in figure 9. First the samples were exposed to the PG plasma plume for $120 \mathrm{~s}$ at different repetition rates. It can be seen that the plasma treatments do not induce any visible damages or burn signs on skin (figure 9 b) compared to the non-treated control (figure 9a), whatever the used conditions, giving thus a good indication of safe skin treatment. Second, to allow monitoring of the distribution of the plasma-generated RONS, which can diffuse all over the surface, samples were soaked two hours in the KI-starch solution before plasma treatment. RONS distribution on the pig ear skin samples is shown in figure $9 \mathrm{~d}$. Pig skin treated at 0.5 and $2 \mathrm{kHz}$ showed a focalized accumulation of RONS while at $20 \mathrm{kHz}$ the reactive species are distributed in a much more larger surface. This confirms the previous results obtained by treating the KI-starch agarose gels (fig.8).

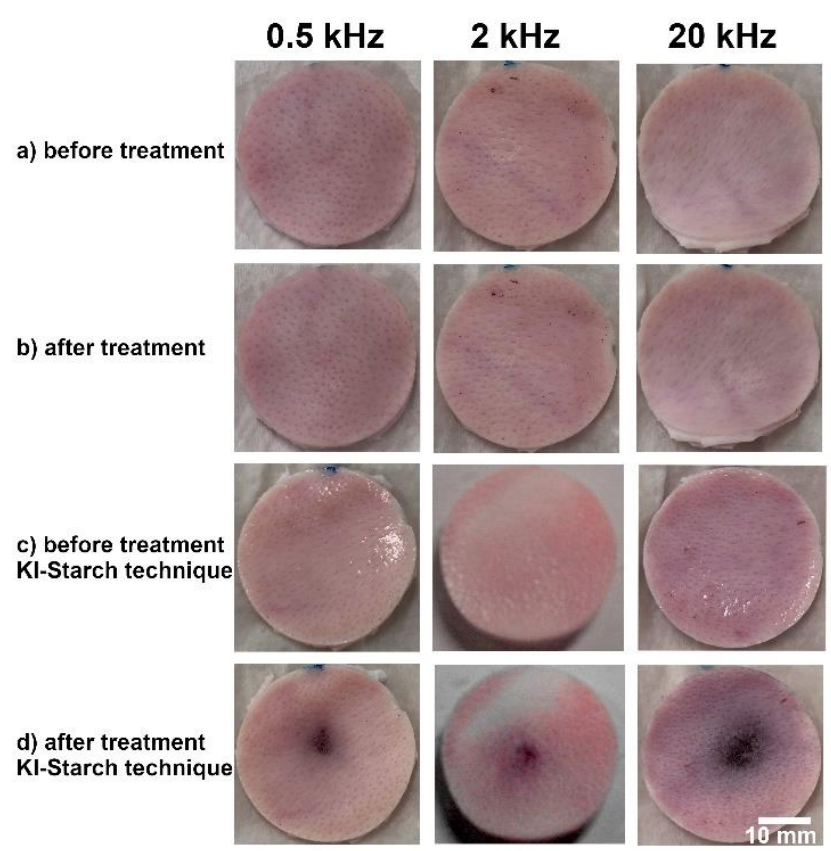

Figure 9. Representative photographs of the pig skin samples a) before or b) after PG treatment of skin, c) before and d) after plasma treatment of KI-starch soaked sample. The treatment time and the gap distance are $120 \mathrm{~s}$ and $15 \mathrm{~mm}$, respectively.

Many of the RONS produced by plasma (e.g., $\mathrm{H}_{2} \mathrm{O}_{2}, \mathrm{NO}^{\bullet}$, $\mathrm{NO}_{2}^{-}, \mathrm{NO}_{3}^{-}$) are also produced in cells and perform biological functions. RONS regulate cellular signaling processes and can stimulate cell growth or trigger cell death. $\mathrm{H}_{2} \mathrm{O}_{2}$ is a quite important and biologically relevant reactive species as well a wound healing mediator. At low concentrations, $\mathrm{H}_{2} \mathrm{O}_{2}$ takes part in intracellular signaling while high $\mathrm{H}_{2} \mathrm{O}_{2}$ concentrations are associated with DNA, lipid and protein damage. Besides, nitrite, was shown to play several biological functions protecting skin from UVA exposure, improving wound healing, vasodilation and neoangiogenesis. -[39, 41].

With the objective to assess RONS penetration across skin for biomedical and cosmetics applications, we first compared the production of hydrogen peroxide and nitrite concentrations upon plasma treatment of DPBS according to the applied frequency. Each well of a 96 well plate filled with $100 \mu \mathrm{l}$ of DPBS was exposed to PG set at $9 \mathrm{kV}, 15 \mathrm{~mm}$ gap, $0.5 \mathrm{slm}$ operative conditions, for different treatment times ranging from 30 to $300 \mathrm{~s}$ for $2 \mathrm{kHz}$ pulse frequency and from 3 to 120 $\mathrm{s}$ for $20 \mathrm{kHz}$ pulse frequency. As shown in figure 10, the nitrite concentrations exhibit a linear dependence versus time and both pulse frequencies ( 2 and $20 \mathrm{kHz}$ ) investigated in this study. The rate increase measured at $20 \mathrm{kHz}$ is about 0.4116 nmol s${ }^{-1}$ that is 3.4 times that measured at $2 \mathrm{kHz}$, this later 
being about of $0.121 \mathrm{nmol} \mathrm{s}^{-1}$. For hydrogen peroxide, a linear increase is observed only for short treatment durations, i.e. up to $150 \mathrm{~s}$ for $2 \mathrm{kHz}$ operation and $30 \mathrm{~s}$ for $20 \mathrm{kHz}$. The regression curve calculated over the linear range, results in a rate increase of $0.13 \mathrm{nmol} \mathrm{s}^{-1}$ and $0.579 \mathrm{nmol} \mathrm{s}^{-1}$ for 2 and 20 $\mathrm{kHz}$ respectively, i.e. 4.5 -fold increase at $20 \mathrm{kHz}$ with respect to the $2 \mathrm{kHz}$ regime. Conversely to the nitrite generation, hydrogen peroxide production exhibits a saturation for the longer treatment durations. Such saturation can be attributed to the quenching of hydrogen peroxide by other RONS delivered in the DPBS solution, the quenching reactions becoming gradually dominant as the hydrogen peroxide concentration and other RONS concentration increase. It is finally measured that the $20 \mathrm{kHz}$ regime, leads to a mean 4 fold RONS rate increase. In our experimental conditions, the ratio of $\mathrm{H}_{2} \mathrm{O}_{2}$ versus $\mathrm{NO}_{2}^{-}$for a $120 \mathrm{~s}$ long treatment are about $35 / 35$ and about $120 / 110$ for respectively $2,20 \mathrm{kHz}$ regimes. This means that for short treatment times such ratio is almost constant.
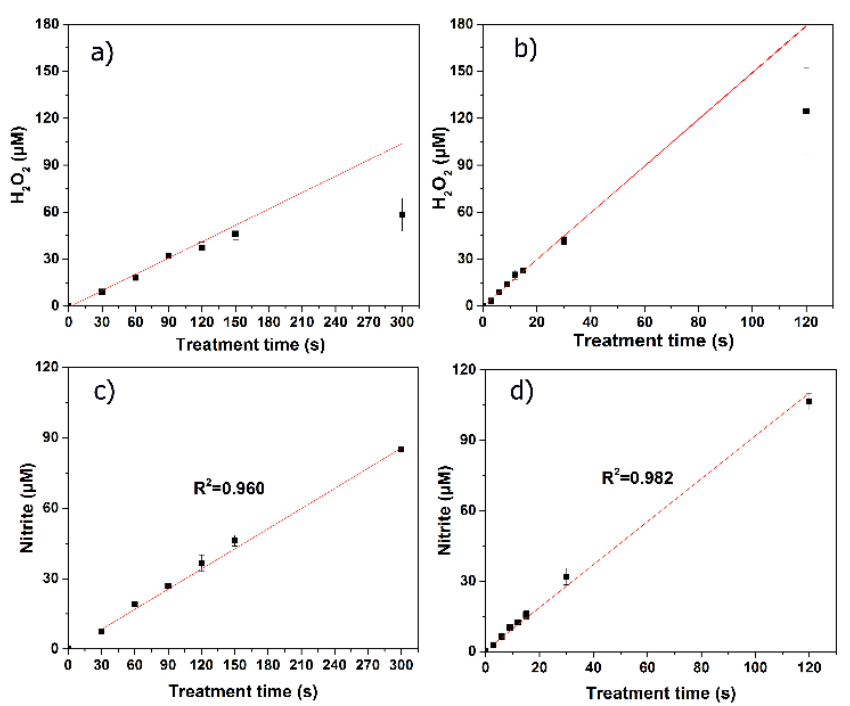

Figure 10. The concentrations of a) $\mathrm{H}_{2} \mathrm{O}_{2}$ at $2 \mathrm{kHz}$, b) $\mathrm{H}_{2} \mathrm{O}_{2}$ at 20 $\mathrm{kHz}, \mathrm{c}$ ) nitrites at $2 \mathrm{kHz}$ and d) nitrites at $20 \mathrm{kHz}$ after direct treatment as function of time. Gap distance: $15 \mathrm{~mm}$.

Controlling the penetration depth of RONS into tissues remains a key issue in applications of plasma in medicine. While difficult to determine their distribution in depth, it is of particular interest to evaluate the quantity that can pass through a certain thickness layer under given treatment conditions. To assess such a penetration using skin models, experiments have been done with $2 \mathrm{~mm}$ thick agarose gel samples. They were placed onto a DPBS solution and treated using PG using the same set of parameters as mentioned previously $(9 \mathrm{kV}, 15 \mathrm{~mm}$ gap, $120 \mathrm{~s})$ for different pulse repetition rates. The concentration of $\mathrm{H}_{2} \mathrm{O}_{2}$ and nitrites measured in DPBS after direct treatment (data from figure 10) and after penetration across the agarose gel are shown in figure
11 (a) and (b). It must first be mentioned that plasma characteristics are undoubtedly different when the plasma plume impinges on DPBS solution or on agar samples covering DPBS solution. While not studied in detail in this work, sample conductivity, capacitance and humidity are some of the parameter influencing the plasma plume and the RONS generation in the gaseous phase over the sample. Target impedance compensation protocols recently reported by Stancampiano et al [42] would be relevant to implement to smooth the influence of target on the plasma characteristics. As observed in figure11 (a) and (b), the concentrations of $\mathrm{H}_{2} \mathrm{O}_{2}$ and nitrites generated directly inside DPBS by a direct treatment increase with the frequency, as discussed in the previous paragraph. Once passing through the agarose gel such species can be quantified in the underneath DPBS. It was previously reported by Szili et al that a significant fraction of RONS may be transferred across quite thick agarose gel samples [24]. In our experimental conditions, after plasma treatment of 2 and $20 \mathrm{kHz}$, the amount of the hydrogen peroxide passing through the gel, are respectively 33 and $45 \%$ of those measured by direct treating the PBS at the same frequency. When DPBS is treated through the agarose sample, 40 and $20 \%$ of the nitrite concentration obtained with direct treatment, are measured 15 minutes after plasma delivery. So, the $20 \mathrm{kHz}$ regime results in the modulation of the $\mathrm{H}_{2} \mathrm{O}_{2} / \mathrm{NO}_{2}{ }^{-}$ ratio when DPBS is exposed through agarose gels. Interestingly, RONS delivery dependence with frequency in both direct or across tissue mimicking sample is far from being intuitive and require detailed analysis before translating any in vitro results to clinical situation.

To deepen the mechanistic analysis of RONS delivery across agarose samples, one additional experiment was performed to assess 1/ the RONS diffusion through the agarose gel during the plasma treatment, and 2/ the RONS delivery by agarose gel following the treatment. To do so, gels were treated for $120 \mathrm{~s}$ at 2 and $20 \mathrm{kHz}$. Immediately after plasma exposure, gels were gently moved on a second plate filled with fresh DPBS. The DPBS collected from the first plate was used to quantify RONS diffusion during and right after the treatment while the DPBS collected 15 minutes after the gel transfer was used to measure the RONS passive diffusion. We found that neither hydrogen peroxide (or very few: $0.042 \mathrm{nmol}$ of $\mathrm{H}_{2} \mathrm{O}_{2}$ for the $20 \mathrm{kHz}$ ) nor nitrites are transferred across the agarose gels following the $120 \mathrm{~s}$ treatment, (figure $11 \mathrm{c}$ and d). This shows that the RONS produced during the treatment are not able to cross the $2 \mathrm{~mm}$ depth agarose gel during the 120s treatment. Conversely, RONS need more time, minutes in this work, to diffuse through the gel. These results suggest that during the plasma treatment the reactive species accumulate and eventually react with the agarose gel and then diffuse across this, following their diffusion coefficients 

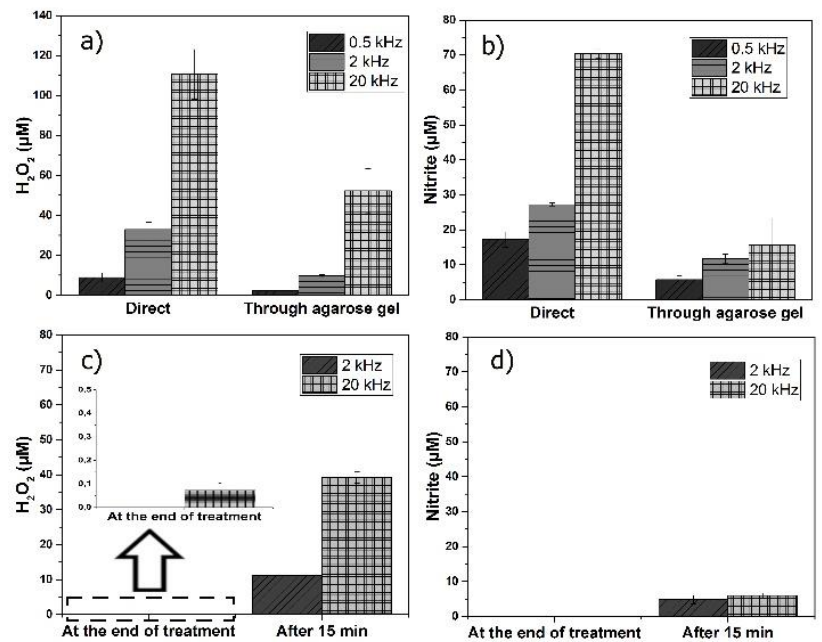

Figure 11. Concentrations of a) $\mathrm{H}_{2} \mathrm{O}_{2}$, b) nitrites after direct treatment and penetration through the agarose gel. For through agarose data, RONS measurement is performed 15 minutes after plasma exposure. The concentration of c) $\mathrm{H}_{2} \mathrm{O}_{2}$ and d) nitrite at the end of the $120 \mathrm{~s}$ plasma treatment and 15 minutes after 120s plasma exposure and gel transfer. Treatment time: $120 \mathrm{~s}$ and Gap distance: $15 \mathrm{~mm}$.

In connection with plasma-cosmetic skin treatment applications but also for any other tissue exposure, it is worth assessing plasma-induced $\mathrm{pH}$ modulation in/on sample, this last being a strong signaling or chemical agent in many biological processes. The external layers of the epidermis possess a mild acidic $\mathrm{pH}$ [43] which has beneficial effects for the organ. In fact, a relatively low $\mathrm{pH}$ preserves the integrity of the skin barrier $[44,45]$, protects the organ from pathogen aggressions $[46,47]$ and promotes the growth of the symbiotic microbiota [48]. Among the plethora of plasma-generated RONS, some species possess acidic characteristics [49] and the lipid surface of biological tissues and of human skin have been shown to be acidified by plasma treatment [50, 51]. All of this shows that it is very important to have a good knowledge of the potential acidification induced by the treatment, a strong decrease of $\mathrm{pH}$ being potentially harmful [51]. Conventional pH measurements on human skin usually rely on flat $\mathrm{pH}$ meter. This apparatus is of good use for Dielectric Barrier discharge (DBD) plasma treatment that creates a relatively large and uniform RONS distribution on the treated surface, but appears less adapted for plasma jet treatments with RONS delivery on a usually more focalized surface, which can be smaller than the probe surface itself. Since the flat $\mathrm{pH}$ meter measures the average of the hydronium concentration underneath the flat probe, the measured acidification can be underestimated. To overcome this limitation, we developed a fluorimetric method that permit not only to measure the $\mathrm{pH}$ but also to visualize it on very small surfaces. In this work we used the protocol of the recently published method [33] to tune both the produced chemically reactive species and the $\mathrm{pH}$ of the tissue. The agarose gel models with a pH of 5.5 mimicking the skin tissue were treated for 120s with PG. According to the figure 12, when the lower frequencies were used $(0.5 \mathrm{kHz}$ and $2 \mathrm{kHz})$, the plasma jet induces a focalized $\mathrm{pH}$ drop. In the center of the sample, which is on the axis of the capillary, the $\mathrm{pH}$ decreases to 4.5 for 2 $\mathrm{kHz}$. By increasing the frequency to $20 \mathrm{kHz}$, the plasma jet led to a more uniform acidification (with a minimum around 4.7) than for $2 \mathrm{kHz}$, more compatible with values for safe human skin treatment.
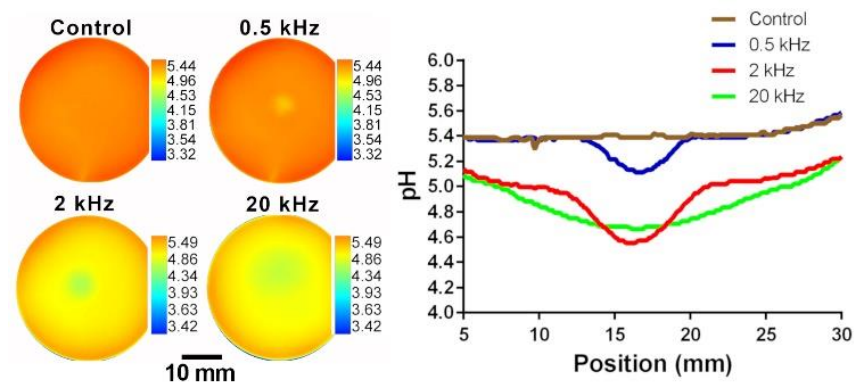

Figure 12. The profiles of $\mathrm{pH}$ variations on agarose gel after the plasma treatment. Treatment time: $120 \mathrm{~s}$ and Gap distance: $15 \mathrm{~mm}$.

\section{Conclusion}

Nowadays, to address the most demanding challenges in biomedicine and in cosmetics, it is essential to develop plasma devices with tightly controlled parameters. This is highly important for further experimental in vitro and in vivo studies of plasmas interactions with biological objects and tissues. In this work, new plasma jet conditions providing a uniform treatment over a large area at atmospheric pressure have been shown

The influence of the gap distance and pulse repetition rate on helium PG plasma discharge characteristics and the induced effects on agarose gel or pig skin samples have been studied. As expected, it has been shown that the current, the charge deposition on the target, as well as the temperature of the target during the treatment and RONS production are very dependent on the gap distance and frequency. But, counterintuitively, the treatment at the higher pulse repetition rate of $20 \mathrm{kHz}$ led to a more uniform and more spread distribution of reactive species. Finally $20 \mathrm{kHz}$ operation is out of the audible frequency range making the treatment more convenient. According to the Schlieren and ICCD imaging, when the frequency is increased to $20 \mathrm{kHz}$, the discharge above and in contact with the target becomes more diffuse and more widely spread. Under such conditions, more air can mix with the helium flow leading not only to an increase in the production but also to a more uniform distribution of the RONS, and enlarging the scale of the downstream surface treatment on agarose gel target. The effects of RONS depend on the location/environment in which they are generated, the 
time they are maintained at that location and most importantly, the concentration at which they are generated. In dermatology, $\mathrm{H}_{2} \mathrm{O}_{2}$ is frequently used as topical antiseptic, it has a haemostatic function and it can induce skin whitening. Nitrites and the derived $\mathrm{NO}^{\bullet}$ were shown to play several physiological activities. $\mathrm{H}_{2} \mathrm{O}_{2}$ and nitrite at relatively low concentrations are beneficial and it is necessary to focus the amount of delivery of RONS to the target. Therefore, this study is important in the context that it addresses the need to explain the RONS concentrations and delivery depth within tissue. The methodology presented in this research can be developed to provide a more quantitative understanding of RONS delivery into skin.

Interestingly, the same effect has been observed on pig ear skin samples. This has also been confirmed by spatially resolved $\mathrm{pH}$ measurements. At $20 \mathrm{kHz}$, the decrease in $\mathrm{pH}$ is rather limited and quite uniform all over the sample. As expected, the increase of pulse frequency, to the $20 \mathrm{kHz}$ regime, allow for a substantial increase in RONS concentration delivery. But RONS delivery in both direct or across tissue mimicking sample treatments and its dependence with pulse frequency are far from being obvious and require detailed analysis before translating any in vitro results to clinical situation. The presented results and observations clearly confirm the importance of the frequency to control the plasma jet characteristics for tissue compatible treatments. With a single plasma jet, without adding nitrogen or oxygen to helium and just by applying the right frequency, we are able to increase, in a uniform way, the treated surface, while providing features interesting for skin and potentially cosmetic treatment.

\section{Acknowledgements}

This work was supported by Cosmetosciences, a global training and research program dedicated to the cosmetic industry, located in the heart of the Cosmetic Valley. This program led by University of Orléans is funded by the Région Centre-Val de Loire, France. Giovanni Busco, Azadeh Valinataj Omran and Loïck Ridou are supported by Cosmetosciences (PLASMACOSM2 Project, Grant 2017 00118114). This work was performed in the frame of the French CNRS networks, GDR2025 HAPPYBIO and GDR3711 Cosmactifs.

\section{References}

[1] Arndt S, Unger P, Wacker E, Shimizu T, Heinlin J, Li Y F, Thomas H M, Morfill G E, Zimmermann J L, Bosserhoff A K and Karrer S 2013 PloS one 8 e 79325

[2] Isbary G, Stolz W, Shimizu T, Monetti R, Bunk W, Schmidt H U, Morfill G E, Klämpfl T G, Steffes B, Thomas H M and Heinlin J 2013 Clin. Plasma Med. 1 25-30
[3] Keidar M, Shashurin A, Volotskova O, Ann Stepp M, Srinivasan P, Sandler A and Trink B 2013 Phys. Plasmas 20057101

[4] Vandamme M, Robert E, Pesnel S, Barbosa E, Dozias S, Sobilo J, Lerondel S, Le Pape A and Pouvesle J M 2010 Plasma Process Polym. 7 264-273

[5] Bauer G, Sersenová D, Graves D B and Machala Z 2019 Sci. Rep. $91-28$

[6] Lu X, Cao Y, Yang P, Xiong Q, Xiong Z, Xian Y and Pan Y 2009 IEEE Trans. Plasma Sci. 37 668-673

[7] Hoffmann C, Berganza C and Zhang J 2013 Med. Gas Res. 321

[8] Ranjan R, Krishnamraju P V, Shankar T and Gowd S 2017 J. Int. Soc. Prev. Community Dent. 771

[9] Gherardi M, Tonini R and Colombo V 2018 Trends biotechnol. 36 583-585

[10] Stoffels E, Sakiyama Y and Graves D B 2008 IEEE Trans. Plasma Sci. 36 1441-1457

[11] Klämpfl T G, Isbary G, Shimizu T, Li Y F, Zimmermann J L, Stolz W, Schlegel J, Morfill G E and Schmidt H U 2012 Appl. Environ. Microbiol. 78 5077-5082

[12] Emmert S, Brehmer F, Hänßle H, Helmke A, Mertens N, Ahmed R, Simon D, Wandke D, Maus-Friedrichs W, Däschlein G and Schön M P 2013 Clin. Plasma Med. 1 24-29

[13] Heinlin J, Morfill G, Landthaler M, Stolz W, Isbary G, Zimmermann J L, Shimizu T and Karrer S 2010 J. Dtsch. Dermatol. Ges. 8 968-976

[14] Busco G, Fasani F, Dozias S, Ridou L, Douat C, Pouvesle J M, Robert E and Grillon C 2017 IEEE Trans. Radiat. Plasma Med. Sci. 2 147-152

[15] Bernhardt T, Semmler M L, Schäfer M, Bekeschus S, Emmert S and Boeckmann L 2019 Oxid. 2019

[16] Kong M G, Kroesen G, Morfill G, Nosenko T, Shimizu T, Van Dijk J and Zimmermann J L 2009 New J. Phys. 11115012

[17] Bekeschus S, Favia P, Robert E and von Woedtke T 2019

Plasma Process Polym. 161800033

[18] Ghasemi M, Olszewski P, Bradley J W and Walsh J L 2013 J. Phys. D: Appl. Phys. 46052001

[19] Foest R, Kindel E, Ohl A, Stieber M and Weltmann K D 2005 Plasma Phys. Control. Fusion 47 B525-B536

[20] Nie Q Y, Cao Z, Ren C S, Wang D Z and Kong M G 2009 New J. Phys. 11115015

[21] Robert E, Darny T, Dozias S, Iseni S and Pouvesle J M 2015 Phys. Plasmas 22122007

[22] Norberg S A, Johnsen E and Kushner M J 2015 Plasma Sources Sci. Technol. 24035026 
[23] Scionti G, Moral M, Toledano M, Osorio R, Duran J D, Alaminos M, Campos A and López-López M T 2014 J. Biomed. Mater. Res. Part A 102 2573-2582

[24] Szili E J, Oh J S, Hong S H, Hatta A and Short R D 2015 J. Phys. D: Appl. Phys. 48202001

[25] Kawasaki T, Sato A, Kusumegi S, Kudo A, Sakanoshita T, Tsurumaru T, Uchida G, Koga K and Shiratani M 2016 Appl. Phys. Express 9076202

[26] Duan J, Lu X and He G 2017 Phys. Plasmas 24073506

[27] Szili E J, Oh JS, Fukuhara H, Bhatia R, Gaur N, Nguyen C K, Hong S H, Ito S, Ogawa K, Kawada C and Shuin T 2018 Plasma Sources Sci. Technol. 27 014001-0140016

[28] Omran A, Busco G, Dozias S, Grillon C, Pouvesle J M and Robert E 2019 Distribution and penetration of reactive oxygen and nitrogen species through a tissue phantom after Plasma Gun treatment 24th Inter. Symposium on Plasma Chemistry

[29] Omran A, Busco G, Ridou L, Dozias S, Grillon C, Pouvesle J M and Robert E 2019 Cold Atmospheric Plasma for safe and tolerable tissue treatment 1 st Inter. Meeting on Plasma Cosmetic Science

[30] Robert E, Sarron V, Ries D, Dozias S, Vandamme M and Pouvesle J M 2012 Plasma Sources Sci. Technol. 21034017

[31] Darny T, Pouvesle J M, Fontane J, Joly L, Dozias S and Robert E 2017 Plasma Sources Sci. Technol. 26105001

[32] Kawasaki T, Mitsugi F, Koga K and Shiratani M 2019 J. Appl. Phys. 125213303

[33] Busco G, Omran A V, Ridou L, Pouvesle J M, Robert E and Grillon C 2019 J. Phys. D: Appl. Phys. 52 24LT01

[34] Fish R M and Geddes L A 2009 Eplasty 9

[35] Suschek C V, Schewe T, Sies H and Kröncke K D 2006 Biol. Chem. 387 499-506

[36] Liebmann J, Born M and Kolb-Bachofen V 2010 J. Invest. Dermatol. 130 259-269

[37] Riès D, Dilecce G, Robert E, Ambrico P F, Dozias S and Pouvesle J M 2014 J. Phys. D: Appl. Phys. 47275401

[38] Morabit Y, Whalley R D, Robert E, Hasan M I and Walsh J L 2019 Plasma Process Polym. e1900217

[39] Graves D B 2012 J. Phys. D: Appl. Phys. 45263001

[40] Rundle RE, Foster J F and Baldwin R R 1944 J. Am. Chem. Soc. 66 2116-2120

[41] Winter J, Tresp H, Hammer M U, Iseni S, Kupsch S, SchmidtBleker A, Wende K, Dünnbier M, Masur K, Weltmann K D and Reuter S 2014 J. Phys. D: Appl. Phys. 47285401.
[42] Stancampiano A, Chung T H, Dozias S, Pouvesle J M, Mir L M and Robert E 2019 IEEE Trans. Radiat. Plasma Med. Sci.

[43] Ali S M and Yosipovitch G 2013 Acta Derm-Venereol. 93 261269

[44] Schmid-Wendtner M H and Korting H C 2006 Skin Pharmacol. Phys. 19 296-302

[45] Angelova-Fischer I, Fischer T W, Abels C and Zillikens D 2018 Br. J. Dermatol. 179 471-477

[46] Schmid M H and Korting H C 1995 Dermatol. 191 276-280

[47] Elias P M 2007 The skin barrier as an innate immune element. In Seminars in immunopathology (Springer-Verlag)

[48] Lambers H, Piessens S, Bloem A, Pronk H and Finkel P 2006 Int. J. Cosmet. Sci. 28 359-370

[49] Chauvin J, Judée F, Yousfi M, Vicendo P and Merbahi N 2017 Sci. Rep. 7 1-15

[50] Helmke A, Hoffmeister D, Mertens N, Emmert S, Schuette J and Vioel W 2009 New J. Phys. 11115025

[51] Heuer K, Hoffmanns M A, Demir E, Baldus S, Volkmar C M, Röhle M, Fuchs P C, Awakowicz P, Suschek C V and Opländer C 2015 Nitric Oxide 44 52-60 